\title{
Turning in Parkinson's Disease Patients and Controls: The Effect of Auditory Cues
}

\author{
Anne-Marie Willems, $\mathrm{PhD},{ }^{1 *}$ Alice Nieuwboer, $\mathrm{PhD},{ }^{1}$ Fabienne Chavret, $\mathrm{MSc},{ }^{1}$ Kaat Desloovere, $\mathrm{PhD},{ }^{1}$ \\ René Dom, MD, ${ }^{2}$ Lynn Rochester, PhD,${ }^{3}$ Gert Kwakkel, PhD,${ }^{4}$ Erwin van Wegen, PhD,${ }^{4}$ and \\ Diana Jones, $\mathrm{PhD}^{3}$ \\ ${ }^{1}$ Department of Rehabilitation Sciences of the Faculty of Rehabilitation Sciences and Physiotherapy, Katholieke Universiteit \\ Leuven, Belgium \\ ${ }^{2}$ Department of Neurosciences of the Faculty of Medicine, Katholieke Universiteit Leuven, Belgium \\ ${ }^{3}$ School of Health, Community and Education Studies, Northumbria University, Newcastle upon Tyne, United Kingdom \\ ${ }^{4}$ Department of Rehabilitation, Medicine, Vrije Universiteit Medisch Centrum, Amsterdam, The Netherlands
}

\begin{abstract}
Turning is an impaired activity in persons with Parkinson's disease (PwPD). The current study examines the turning characteristics in PwPD (9 freezers and 10 nonfreezers) and 9 controls, and explores the effect of rhythmic auditory cues while turning. Turning parameters were collected from a $180^{\circ}$ left U-turn during a noncued and a cued condition, using a 3D measuring system. Auditory cues were supplied with a metronome at a rhythm equaling the subject's comfortable step frequency during straight line walking. Results showed that in contrast to controls, PwPD used a wider turning-arc and took smaller, narrower steps. In addition, they demonstrated a higher
\end{abstract}

Coefficient of Variation (CV) of step duration (6.92\%) compared to controls $(4.88 \%, P<0.05)$. The "wide-arc" turning strategy of PwPD was more prominent in freezers than in nonfreezers. Auditory cues reduced the CV of step duration in PwPD (both freezers and nonfreezers) during turning (from 6.92 to $6.00 \%, P<0.05)$. In summary: Cueing reduced the gait-timing variability during turning, but PwPD maintained a wider arc to turn compared with controls. (C) 2007 Movement Disorder Society

Key words: Parkinson's disease; gait; turning; freezing; cueing
Most studies investigating gait in people with Parkinson's disease (PwPD) concentrate on walking in a straight line ${ }^{1}$ although turning is often impaired. ${ }^{2}$ Turning performance can be affected by impaired trunk mobility, ${ }^{2-5}$ difficulties coordinating the turn, ${ }^{6}$ freezing, ${ }^{7-9}$ and postural instability or falls. ${ }^{10,11}$ In PwPD, the range of trunk movement is diminished ${ }^{3}$ and everyday trunk rotations are impaired. ${ }^{2,4}$ In addition, the "en bloc" movement of the head-shoulder unit in parkinsonian gait $^{5}$ may hinder normal dissociated rotation while turning, ${ }^{12}$ suggesting that PwPD encounter difficulties coordinating these simultaneous movements. ${ }^{6}$

*Correspondence to: Dr. Anne-Marie Willems, K.U. Leuven, Department of Rehabilitation Sciences, Tervuursevest 1013001 Heverlee, Belgium. E-mail: annemarie.willems@gmail.com

Received 1 November 2006; revised 17 January 2007; accepted 27 January 2007

Published online 26 June 2007 in Wiley InterScience (www. interscience.wiley.com). DOI: 10.1002/mds.21445
Freezing, typically described as "involuntary and sudden cessation of walking," is a common problem in advanced Parkinson's disease. ${ }^{8}$ Giladi et al., reported that from a sample of 318 PwPD who experienced freezing, $45 \%$ reported freezing while turning. ${ }^{9}$ The association of turning with freezing and falls prompts research to focus on ways to increase turning stability and safety. ${ }^{7-9,11}$ During normal gait, PwPD who freeze ${ }^{13,14}$ and fall ${ }^{15}$ have increased gait-timing variability, which may be a measurable precursor of falling and freezing. It has been suggested that an impaired internal timekeeping mechanism may underlie increased gait-timing variability. ${ }^{15,16}$ An auditory cue may provide an external rhythm to counteract this problem. Del Olmo et al., demonstrated a reduction in gait-timing variability in PwPD after 4 weeks of training with auditory cues. ${ }^{17}$ In addition, a recent study showed that auditory cues had a positive effect on spatiotemporal gait parameters during straight 
TABLE 1. Subject characteristics

\begin{tabular}{|c|c|c|c|c|c|c|c|c|}
\hline & Age (years) & Weight (kg) & Height $(\mathrm{cm})$ & MMSE & DD (years) & FoGQ & UPDRS III & $\mathrm{H} \& \mathrm{Y}$ \\
\hline Controls & 62.6 & 72.3 & 162.7 & 28.9 & & & & \\
\hline$S D$ & 3.9 & 8.5 & 5.7 & 0.8 & & & & \\
\hline Nonfreezers & $60.6 *$ & 71.5 & 168.5 & 28.5 & $6.2 *$ & $5.5 * *$ & 24.7 & 2.6 \\
\hline$S D$ & 6.2 & 12.3 & 6.9 & 2.2 & 3.0 & 4.9 & 12.6 & 0.7 \\
\hline Freezers & 68.1 & 65.0 & 163.4 & 26.9 & 11.5 & 15.6 & 27.9 & 2.8 \\
\hline$S D$ & 7.3 & 8.3 & 5.6 & 2.2 & 6.0 & 4.2 & 11.8 & 0.7 \\
\hline
\end{tabular}

The mean and standard deviation (SD) are presented.

*Freezers and nonfreezers significantly different at $P<0.05$ ( $t$-test).

**Freezers and nonfreezers statistically different at $P<0.01$ ( $t$-test).

DD: Disease duration, FoGQ: Freezing of gait questionnaire.

line walking in PwPD. However, different cueing frequencies were required for freezers and nonfreezers. ${ }^{18}$

The present study had three aims: to examine differences in the spatiotemporal turning parameters in PwPD and controls; to examine the effect of auditory cueing on these parameters and to evaluate the difference between freezers and nonfreezers.

\section{METHODS}

\section{Subjects}

Nineteen PwPD and 9 healthy age-matched controls were recruited. Patients were referred by their neurologist and were included if they met following inclusion criteria: diagnosis of idiopathic Parkinson's disease (stage I-IV in on-phase on modified Hoehn and Yahr scale $^{19}$ ); able to walk for $10 \mathrm{~m}$ repeatedly and no unpredictable off-phases during the test. They were excluded if they had: comorbidity limiting gait; undergone functional brain surgery; hearing deficits; severe dyskinesias during the test (score $>1$ Modified Dyskinesia Scale) ${ }^{20}$; dementia (score $\leq 24$ Mini Mental State Examination, MMSE) $^{21}$ (See Table 1). Patients were divided into freezers (9) and nonfreezers (10) using the Freezing of Gait questionnaire (FoGQ). ${ }^{22}$ Freezers were defined as PwPD who experienced freezing episodes at least once a week. All subjects gave their written informed consent according to the Helsinki Declaration and ethical approval was received from the local ethics committee.

\section{Test-Protocol}

The protocol required subjects to walk along a walkway on which an obstacle was placed at a standard distance of $5 \mathrm{~m}$, make a left turn $\left(180^{\circ}\right)$ around it and return to their starting position. The dimensions of the obstacle were such that subjects were required to turn through an arc (multi-step U-turn). Subjects performed the turn under two conditions:
1. Noncued: "Walk towards the obstacle, make a left turn around it and return to your starting position. Please walk at your normal, comfortable speed.

2. Cued: "Walk towards the obstacle, make a left turn around it and return to your starting position. Try to synchronize every foot-contact with the beat of the metronome. Follow the cues while walking and turning."

Each condition was repeated three times and conditions were performed in the order described above to prevent carry-over from cued to noncued turn. During the cued turn, auditory cues were delivered via a metronome throughout the entire trial. Cues were delivered at the preferred step frequency of straight line walking, determined from the middle section of the walkway (4-6 steps on average). Measurements were taken in on-phase approximately $1 \mathrm{hr}$ after medication intake.

\section{Apparatus}

Gait analysis was performed using an eight camera VICON data capturing system positioned around an eight meter walkway (Vicon Motion Systems, workstation 612 with lower limb plug-in gait marker placement). Prior to each analysis, the measurement area was calibrated. Retro-reflective markers (14 $\mathrm{mm}$ in diameter) were placed on the sacrum and bilaterally on the anterior superior iliac spine, the mid-thigh, the lateral femoral condyle, the mid-shank, the lateral malleolus, the dorsal aspect of the foot between the second and third metatarsal head, and on the calcaneus. The turning obstacle was a cube-shaped frame made of plastic pipes (height $40 \mathrm{~cm}$, width $40 \mathrm{~cm}$, length $60 \mathrm{~cm}$ ). Two retro-reflective markers were placed on the furthermost two corners of the obstacle to determine the exact position of those markers within the horizontal plane of the measurement area (XY-coordinates). The Y-axis was the vertical axis, corresponding to the line of progression, whereas the $\mathrm{X}$-axis 
Turning parameters used in analysis, demonstrated on a trial of a control subject.

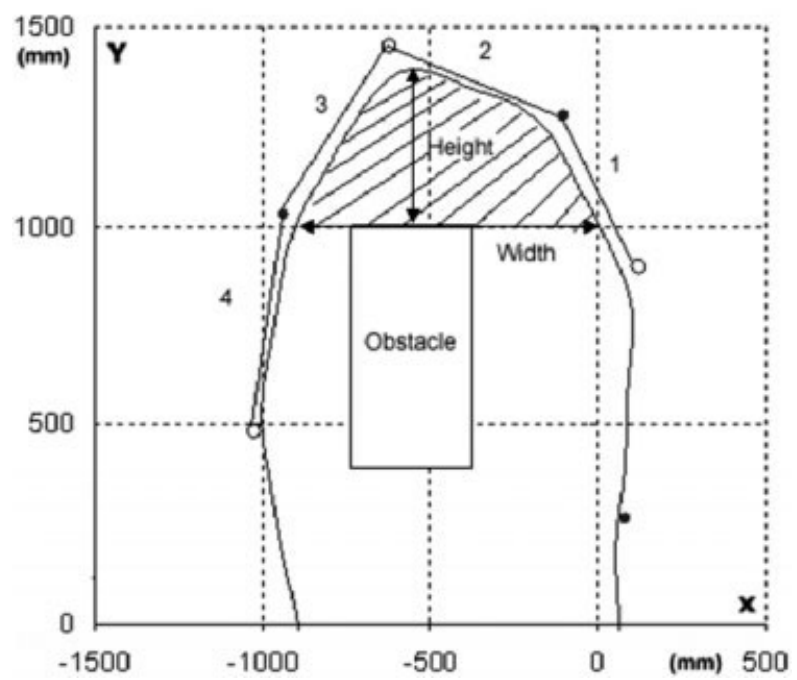

FIG. 1. Sacrum displacements are presented by the solid curve. The height and width of the turning-arc are indicated. Solid dots represent the position of the left calcaneus at initial contact, open dots represent initial contact of the right foot. This subject needed 4 steps to complete the turn.

was the horizontal axis. The Y-coordinates of the obstacle equaled 1,000 mm (See Fig. 1).

\section{Data Processing}

The turn was described by three groups of variables: general turning parameters; step parameters and gaittiming variability (See Fig. 1).

(1) General turning parameters: i.e., turn time, shape of the turning-arc, and number of steps to turn. The beginning and end of the turn were defined by the sacrum passing the furthermost corners of the turning obstacle (sacrum Y-coordinate equaling 1,000 $\mathrm{mm}$ ). The time between start and end point is the turn time. The height of the turning-arc is the largest value of the sacrum Y-coordinate. The width of the turning-arc (X-axis) is the distance between the start and end of the turn where the $\mathrm{Y}$-coordinate equals $1,000 \mathrm{~mm}$. The turning-arc is the distance between the XY-coordinates with reference to the sacrum marker calculated with the Pythagoras theorem using all extracted XY-coordinates comprised between the beginning and end of the turn.

(2) Step parameters: i.e., step length, width, and duration for the left and right leg separately. For each step corresponding XY-coordinates for the calcaneus were extracted at the time of initial foot contact. Steps were identified as turning steps rather than straight line walking steps when the Y-coordinates of beginning or end of the step were greater than $1,000 \mathrm{~mm}$ (Fig. 1). Step duration was defined as the time between initial foot contact of one side and initial foot contact of the other side. To obtain the width and length of turning steps, the calculation for nonlinear walking proposed by Huxham et al. ${ }^{23}$ was used (Appendix A). Data were analyzed using Bodybuilder (to extract XY-coordinates and define marker displacements) and Workstation and Polygon software (to define initial foot contacts) from Oxford Metrics, Oxford, UK.

(3) Gait-timing variability: The Coefficient of Variation (CV) of step duration was calculated as the standard deviation (SDEV), expressed as a percentage of the mean $(\mathrm{CV}=100 * \mathrm{SDEV} /$ mean $)$ for the maximum number of turning steps for each patient.

Turning parameters, step parameters, and gait-timing variability were averaged for the 3 walking trials in both turning conditions. Six trials were eliminated due to an incomplete data-set.

\section{Statistical Analysis}

Differences between PwPD and controls and between freezers and nonfreezers for descriptive variables were examined using unpaired $t$-tests or nonparametric tests. Paired $t$-tests were used to compare left-right step values for step duration, step length and step width for each subject group separately in the noncued condition.

A multivariate model of repeated measures was applied to study the effect of cueing, within and between groups using an unstructured or more simplified covariance matrix in SAS (statistical analysis software version 8.02). The effect of cueing as an independent variable was examined on the following outcome variables: number of steps, time to turn, height, width and length of the turning-arc, step length left and right, step width left and right, step duration left and right and the $\mathrm{CV}$ of step duration. Two group comparisons were carried out: PwPD versus controls and freezers versus nonfreezers. Results from this analysis are reported as estimated means and standard errors. Considering the small size of subject groups no correction for group differences was carried out. Significance levels for all tests were set at 0.05 .

\section{RESULTS}

Patients and controls were comparable for all subject characteristics (Table 1). Freezers were significantly older, experienced longer disease duration and had a higher FoGQ-score than nonfreezers. Table 2 presents normal turning trials in all subjects. One patient froze 
TABLE 2. Turning parameters for the noncued turn

\begin{tabular}{|c|c|c|c|c|c|c|c|c|c|c|}
\hline \multirow[b]{2}{*}{ Parameter } & \multicolumn{2}{|c|}{ Control (9) } & \multicolumn{2}{|c|}{ Parkinson (19) } & \multirow[b]{2}{*}{$P$-value } & \multicolumn{2}{|c|}{ Nonfreezers (10) } & \multicolumn{2}{|c|}{ Freezers (9) } & \multirow[b]{2}{*}{$P$-value } \\
\hline & $\begin{array}{c}\text { Estimated } \\
\text { mean }\end{array}$ & SE & $\begin{array}{l}\text { Estimated } \\
\text { mean }\end{array}$ & SE & & $\begin{array}{c}\text { Estimated } \\
\text { mean }\end{array}$ & SE & $\begin{array}{c}\text { Estimated } \\
\text { mean }\end{array}$ & SE & \\
\hline Steps & 4.0 & 0.1 & 5.4 & 0.2 & $<0.0001$ & 5.2 & 0.3 & 5.9 & 0.4 & 0.1464 \\
\hline Time (sec) & 1.6 & 0.1 & 2.6 & 0.2 & $<0.0001$ & 2.4 & 0.2 & 2.8 & 0.2 & 0.2485 \\
\hline Height (mm) & 1412.3 & 19.9 & 1476.9 & 18.8 & 0.0164 & 1440.5 & 19.7 & 1517.3 & 27.5 & 0.0313 \\
\hline Width (mm) & 522.2 & 27.2 & 612.3 & 21.6 & 0.0128 & 589.4 & 28.2 & 637.7 & 31.1 & 0.2595 \\
\hline Length (mm) & 1334.7 & 47.5 & 1510.9 & 48.3 & 0.0147 & 1422.0 & 55.1 & 1609.7 & 67.6 & 0.0402 \\
\hline StepL L (mm) & 419.0 & 29.3 & 319.5 & 17.5 & 0.0065 & 321.1 & 23.8 & 317.1 & 25.7 & 0.8994 \\
\hline Step L R (mm) & 498.1 & 12.9 & 393.6 & 17.4 & $<0.0001$ & 396.1 & 26.2 & 390.9 & 22.4 & 0.8812 \\
\hline Step W L (mm) & 255.7 & 13.0 & 184.8 & 11.4 & 0.0003 & 184.2 & 15.4 & 185.5 & 16.7 & 0.9569 \\
\hline Step W R (mm) & 107.1 & 12.9 & 56.2 & 8.6 & 0.0026 & 59.0 & 13.9 & 53.0 & 9.4 & 0.7248 \\
\hline Step D L (sec) & 0.54 & 0.02 & 0.57 & 0.02 & 0.3181 & 0.57 & 0.02 & 0.57 & 0.03 & 0.7939 \\
\hline Step D R (sec) & 0.55 & 0.02 & 0.60 & 0.02 & 0.0544 & 0.60 & 0.02 & 0.61 & 0.04 & 0.8631 \\
\hline CV sd (\%) & 4.88 & 0.48 & 6.92 & 0.60 & 0.0104 & 6.55 & 0.95 & 7.32 & 0.67 & 0.5110 \\
\hline
\end{tabular}

The estimated mean and the Standard Error (SE) are presented.

Parameters are: the number of steps (steps), the time to turn (time), the height, width and length of the turning-arc (height, width and length), step length left and right (step L L/R), step width left and right (step W L/R), step duration left and right (step D L/R) and the CV of the step duration (CV sd).

Exact $P$-values are presented for group differences: controls and PwPD; nonfreezers and freezers.

during one trial, which is reported separately in Appen$\operatorname{dix} B$.

\section{Noncued Turn}

Patients needed more time and took more steps than controls (Exact $P$-values, see Table 2). In addition, the size of the turning-arc (width, height, and length) was greater in PwPD. Step length was smaller and reduced in width compared to controls for both the right and left foot. There were no statistical significant differences for step duration between PwPD and controls on the right and left side. The CV of step duration was significantly larger in PwPD than in controls. In PwPD the right side step duration was significantly larger than the left. For both PwPD and controls, right step lengths were larger and right step widths were smaller than left sided values.

When comparing the subgroups, the height and length of the turning-arc was increased in freezers compared to nonfreezers but no other differences were observed.

\section{Cued Turn}

There was a similar pattern of results for the cued turn, where most of the group differences remained (Exact $P$-values, see Table 3 ). Patients took more time and more steps to turn; the size of the turning-arc was greater; and turning steps were smaller and narrower with respect to controls. No differences were found for the step duration (left and right) or for the CV of step duration. The freezer and nonfreezer sub-group did not differ for any of the variables.

\section{The Effect of Cueing}

The interaction of group by condition (cueing) was not significant for any of the parameters, but within group analysis demonstrated some changes. The CV of step duration was reduced with cues in PwPD $(P=0.0361)$. No changes were observed for the other spatiotemporal parameters as a result of cueing. Cues did not result in differences in turning parameters for the freezers. Nonfreezers, however, took more steps to complete the turn with cues $(P=0.0270)$ and the height and length of the turning-arc were increased as a result of cues $(P=0.0135$ and $P=0.0033$, respectively).

\section{DISCUSSION}

\section{Turning Characteristics in PD and Controls}

The turning pattern observed for PwPD in turning time, step number, size and width may result from a carry-over effect from slow gait with small steps ${ }^{18}$ to slow turning with many small steps. However, PwPD also needed more space to turn than controls. This "widearc" strategy may be induced by an inability to turn in the same way as controls or to prevent falls or freezing. Recent study confirmed that multi-step turns result in greater stability as the center of gravity remains between the two feet ${ }^{24}$ and therefore multi-step turns may be a compensatory strategy for postural instability in elderly and PwPD ${ }^{11,25}$ Multi-step turning has been recommended for PwPD with postural instability, instead of attempting to "normalize " turning in rehabilitation. ${ }^{11,26}$ 
TABLE 3. Turning parameters for the cued turn

\begin{tabular}{|c|c|c|c|c|c|c|c|c|c|c|}
\hline \multirow[b]{2}{*}{ Parameter } & \multicolumn{2}{|c|}{ Control (9) } & \multicolumn{2}{|c|}{ Parkinson (19) } & \multirow[b]{2}{*}{$P$-value } & \multicolumn{2}{|c|}{ Nonfreezers (10) } & \multicolumn{2}{|c|}{ Freezers (9) } & \multirow[b]{2}{*}{$P$-value } \\
\hline & $\begin{array}{c}\text { Estimated } \\
\text { mean }\end{array}$ & $\mathrm{SE}$ & $\begin{array}{c}\text { Estimated } \\
\text { mean }\end{array}$ & $\mathrm{SE}$ & & $\begin{array}{c}\text { Estimated } \\
\text { mean }\end{array}$ & $\mathrm{SE}$ & $\begin{array}{c}\text { Estimated } \\
\text { mean }\end{array}$ & $\mathrm{SE}$ & \\
\hline Steps & 3.9 & 0.2 & 5.7 & 0.3 & $<0.0001$ & $5.6^{*}$ & 0.4 & 5.8 & 0.5 & 0.7117 \\
\hline Time (sec) & 1.6 & 0.1 & 2.7 & 0.2 & $<0.0001$ & 2.7 & 0.3 & 2.7 & 0.2 & 0.8485 \\
\hline Height (mm) & 1397.2 & 25.8 & 1492.9 & 22.1 & 0.0080 & $1477.5^{*}$ & 31.2 & 1510.0 & 30.3 & 0.4615 \\
\hline Width (mm) & 529.8 & 32.6 & 620.8 & 23.2 & 0.0306 & 599.7 & 27.8 & 644.3 & 36.3 & 0.3372 \\
\hline Length (mm) & 1296.5 & 51.4 & 1546.2 & 50.9 & 0.0016 & $1510.9^{* *}$ & 68.5 & 1585.5 & 73.8 & 0.4648 \\
\hline Step L L (mm) & 438.2 & 24.9 & 314.0 & 20.0 & 0.0006 & 314.9 & 29.0 & 313.0 & 27.2 & 0.9614 \\
\hline Step L R (mm) & 472.7 & 22.1 & 386.6 & 16.6 & 0.0041 & 385.4 & 25.7 & 387.8 & 20.2 & 0.9422 \\
\hline Step W L (mm) & 245.7 & 19.4 & 182.8 & 10.7 & 0.0085 & 180.6 & 14.5 & 185.3 & 15.8 & 0.8257 \\
\hline Step W R (mm) & 129.3 & 16.2 & 52.3 & 7.5 & 0.0002 & 58.2 & 11.6 & 45.8 & 8.7 & 0.4016 \\
\hline Step D L (sec) & 0.52 & 0.02 & 0.57 & 0.02 & 0.0578 & 0.57 & 0.02 & 0.56 & 0.03 & 0.7471 \\
\hline Step D R (sec) & 0.55 & 0.01 & 0.59 & 0.02 & 0.0998 & 0.60 & 0.02 & 0.59 & 0.04 & 0.8295 \\
\hline $\mathrm{CV}$ sd $(\%)$ & 4.98 & 0.61 & $6.00 *$ & 0.41 & 0.1675 & 5.73 & 0.57 & 6.30 & 0.59 & 0.4946 \\
\hline
\end{tabular}

The estimated mean and the Standard Error (SE) are presented.

Parameters are: the amount of steps (steps), the time to turn (time), the height, weight, and length of the turning-arc (height, width, and length), step length left and right (step L L/R), step width left and right (step W L/R), step duration left and right (step D L/R), and the CV of the step duration (CV sd).

Exact $P$-values are presented for group differences: controls and PwPD; nonfreezers and freezers.

*Statistically different from the noncued turn $(P<0.05)$.

** Statistically different from the noncued condition $(P<0.01)$.

The gradual directional change implied by a "wide-arc" turn could also reduce the complexity of the task, in terms of coordinating simultaneous and sequential movements. ${ }^{6}$ The $\mathrm{CV}$ of step duration was significantly higher in PwPD (6.92\%) than in controls (4.88\%). This is in agreement with others who found increased gait-timing variability for PwPD. ${ }^{27,28}$ Gait-timing has been proposed to be regulated automatically by the basal ganglia and mediated by executive functioning, ${ }^{29,30}$ which are both impaired in PwPD. ${ }^{31}$ Basal ganglia dysfunction may turn automated tasks into attention demanding tasks, possibly overloading the executive function capacity. These mechanisms may also be at play during turning.

\section{The Effect of Cues on Turning Parameters}

Auditory cues reduced gait-timing variability in PwPD from $6.92 \%$ to $6.00 \%$. This may be an important clinical finding as increased gait-timing variability was found to be a precursor of falling ${ }^{15}$ and freezing ${ }^{13}$. A larger reduction $(2.19 \%)$ in gait-timing variability of straight line walking was reported after a 4 week training program with auditory cues. ${ }^{17}$ The immediate effect of cueing on variability in the current study may thus increase after cue-training. The immediate influence of cues on motor performance in PwPD has been explained by the activation of an alternative neural circuit. ${ }^{32}$ Lateral pathways are relatively more active, in externally cued motor performance in contrast to medial pathways in automatic motor control. ${ }^{33}$ Whether these mechanisms apply to improvements seen in gait-timing variability (i.e. temporal stability) remains to be determined both in straight line walking and in turning.

\section{Turning Characteristics in Freezers and Nonfreezers}

As expected, freezers demonstrated longer disease duration ${ }^{34}$ and were older than nonfreezers. Disease severity on the other hand was similar which justifies a subgroup comparison. When comparing the three subgroups during the noncued turn, freezers differed mostly from the controls. In contrast to nonfreezers, freezers' turns were larger as both the height and length of the turningarc were increased. These results show that particularly freezers used the "wide-arc" strategy spontaneously. Freezers are expected to find more potential danger in a turning task due to its association with freezing. Plotnik et al., showed that freezers have increased swing time asymmetry during normal walking. ${ }^{35}$ They hypothesized that disturbed motor control of bilateral coordination may be a causal factor of freezing, particularly when performing a turn. The self-chosen "wide-arc" strategy may reduce left-right asymmetry and as such reduce freezing.

Although the CV of step duration was larger in freezers $(7.32 \%)$ than in nonfreezers $(6.55 \%)$, this difference was not significant. This finding is not in agreement with Hausdorff et al., who previously reported an increased gait-timing variability in freezers when compared to non- 
freezers both in the on- and off-phase of the medication cycle during straight line walking. ${ }^{13}$

Both freezers and nonfreezers improved their gaittiming variability under the influence of auditory cueing (from $7.32 \%$ to $6.30 \%$ in freezers, from $6.55 \%$ to $5.73 \%$ in nonfreezers), but this improvement was not significant. For the other turning parameters, the two subgroups differed in their reaction to the cues. Cues drove nonfreezers towards the "wide-arc" strategy as spontaneously present in freezers. Freezers' turn did not change with cues, possibly because they already used this "widearc" strategy. Alternatively, these results may indicate that freezers have less benefit from cueing during turning. A recent study by Cubo et al. showed that auditory cues slowed down walking speed without affecting the number and duration of freezing episodes during the on-phase of walking in freezers. ${ }^{36}$ Our own work, confirms that freezers and nonfreezers show differential spatiotemporal responses to varying cueing frequencies, possibly reflecting different motor control strategies in relation to the use of external cues. ${ }^{18}$

\section{Limitations and Conclusions}

To ensure a reasonable amount of steps to calculate gait-stability, step rather than stride parameters are used. Another advantage of using step parameters is its relative independency from walking speed. ${ }^{27}$ Future work should compare different gait-stability measures (using step and stride parameters) during straight line walking and turning.

Caution is warranted when interpreting the results of the current study for the lack of Bonferroni correction and statistical correction for confounders such as age and disease severity due to the small sample size Future studies should address the influence of disease-dominance on turning characteristics by asking patients to turn to both sides.

In the current study, cueing was set at the individual's preferred step frequency during straight line walking. Follow-up studies need to address which cueing frequency would be most effective in influencing spatiotemporal parameters, turning stability and the risk of freezing. Possibly, optimal cueing for freezers implies a reduction of the stepping rhythm, as it may enlarge step length ${ }^{18}$ and therefore reduce festination.

In conclusion, this study demonstrated that PwPD turn differently from controls. They make a wider turn, while using smaller steps, a strategy which is also prominent in freezers when compared to nonfreezers. In addition, PwPD demonstrate a higher gait-timing variability. Auditory cues reduced the CV of step duration in PwPD (both freezers and nonfreezers) during turning. Cueing

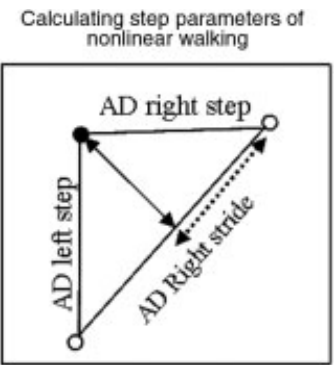

FIG. 2. Calculating step parameters of nonlinear walking The solid dot represent the position of the left calcaneus at initial contact, the open dots represent initial contact of the right foot. The step length right is presented by the dotted arrow, whereas the solid arrow represents the width of the right step.

did not move other turning parameters towards normal values. Further study is needed to understand the effects of different cue settings on different turning conditions before the outcome measures for clinical studies will be clarified.

\section{APPENDIX A DETERMINING STEP PARAMETERS OF THE TURN}

The calculation of step parameters of the turn was based on an algorithm proposed by Huxham et al. ${ }^{28}$ This algorithm requires the determination of the absolute distance (AD) of both steps and strides. For the current experiment, these ADs were calculated by applying the Pythagoras' theorem on the XY-coordinates of the calcanei. In the triangle consisting of the $\mathrm{AD}$ of the right stride (longest side) and the $\mathrm{AD}$ of successively left and right step (see Fig. 2) the formula is the following: (1) Step length right $=\left[(\mathrm{AD} \text { right step })^{2}+(\mathrm{AD}\right.$ right stride $\left.)^{2}-(\mathrm{AD} \text { left step })^{2}\right] / 2 \mathrm{AD}$ right stride. (2) Step width right $=$ Square root of $\left[\mathrm{AD}\right.$ right $\mathrm{step}^{2}-$ step length right $\left.{ }^{2}\right]$.

\section{APPENDIX B FREEZING TRIAL}

One of the freezers showed a freezing episode during the turning trials. This episode occurred at the end of the turn in the first trial of the noncued turn. The subject appeared to be unable to make a right step (Fig. 3). Detailed analysis with the Vicon software showed that the right calcaneus was lifted from the ground and repositioned, but that the subject failed to lift the right foot completely. On the graph, it can be observed that two right foot strikes were positioned closely together (5 $\mathrm{cm}$ apart) at the moment of freezing. When comparing the step lengths when entering the turn with the steps just prior to the freezing episode, a reduction of step length can be noticed. This is in agreement with earlier findings in straight line walking. ${ }^{14}$ The sacrum displacements found in this trial showed an oscillating pattern, similar to that of the center of mass (COM) displacements described for a slow $360^{\circ}$ turn. ${ }^{37}$ It can be observed that the subject used more steps (12), needed more time $(4.8 \mathrm{sec})$, had smaller step lengths both left $(157.4 \mathrm{~mm})$ and right $(163.2 \mathrm{~mm})$, and shorter step duration left $(0.418)$ and right $(0.453)$ than the average values in the noncued and cued condition of the same subject. Moreover, in comparison to the freezer subgroup in the noncued turn (Table 2), general turning parameters and gait timing variability were larger then the freezers' averages whereas step parameters were smaller. No statistics were performed as it is an anecdotal finding. 
Graphical presentation and turning parameters of freezing trial

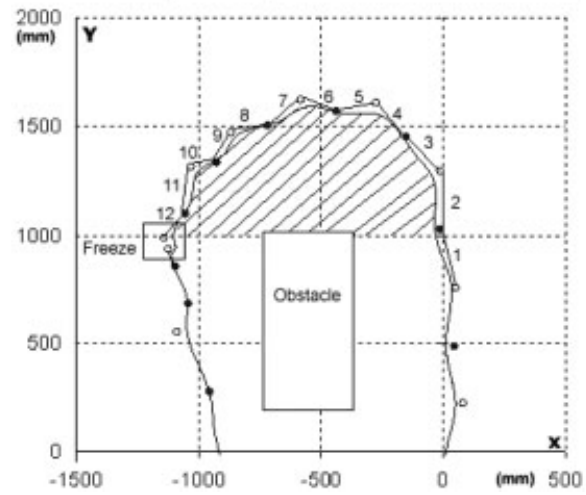

\begin{tabular}{lrrr}
\hline Parameter & Freeze & Non-cued & Cued \\
Steps & 12 & 8.5 & 9.7 \\
Time (sec) & 4.8 & 3.7 & 4.2 \\
Height $(\mathrm{mm})$ & 1592.6 & 1597.4 & 1703.6 \\
Width $(\mathrm{mm})$ & 708 & 787.6 & 825.7 \\
Length $(\mathrm{mm})$ & 1789.4 & 1876.5 & 2115.9 \\
StepL L $(\mathrm{mm})$ & 157.4 & 230 & 243.5 \\
StepL R mm) & 163.2 & 271.7 & 286.9 \\
StepW L (mm) & 79.6 & 86.1 & 96.4 \\
StepW R (mm) & 37.3 & 17.4 & 19.6 \\
StepD L (sec) & 0.42 & 0.47 & 0.48 \\
StepD R (sec) & 0.45 & 0.51 & 0.53 \\
CV sd (\%) & 7.61 & 6.3 & 7.87 \\
\hline
\end{tabular}

FIG. 3. In the table, turning parameters of the freezing trial are presented next to mean values of the remaining noncued and cued trials of the same subject. $\mathrm{Pa}$ rameters are: the amount of steps (steps), the time to turn (time), the height, weight, and length of the turning-arc (height, width, and length), step length left and right (step L L/R), step width left and right (step W L/R), step duration left and right (step D L/R) and the $\mathrm{CV}$ of the step duration ( $\mathrm{CV}$ sd). The square shape indicates where two right foot strikes were positioned closely together $(5 \mathrm{~cm}$ apart), i.e. where freezing occurred.
Acknowledgments: Financial support from the European Commission. Framework V funding; QLRT-2001-00120. RESCUE-project.

\section{REFERENCES}

1. Morris ME, Huxham F, McGinley J, Dodd K, Ianesk R. The biomechanics and motor control of gait in Parkinson disease. Clin Biomech 2001;16:459-470.

2. Nieuwboer A, De Weerdt W, Dom R, Lesaffre E. A frequency and correlation analysis of motor deficits in Parkinson patients. Disabil Rehabil 1998;20:142-150.

3. Shenckman ML, Clark K, Xie T, Kuchibhatla M, Shinberg M, Ray L. Spinal movement and performance of a standing reach task in participants with and without Parkinson's disease. Phys Ther 2001; 81:1400-1411.

4. Steiger MJ, Thompson PD, Marsden CD. Disordered axial movement in Parkinson's disease. J Neurol Neurosurg Psychiatry 1996; 61:645-648.

5. Mesure S, Azulay JP, Pouget J, Amblard B. Strategies of segmental stabilization during gait in Parkinson's disease. Exp Brain Res 1999;129:573-581.

6. Vaugoyeau M, Viallet F, Mesure S, Massion J. Coordination of axial rotation and step execution: deficits in Parkinson's disease. Gait Posture 2003;18:150-157.

7. Schaafsma JD, Balash Y, Gurevich T, Bartels AL, Hausdorff JM, Giladi N. Characterization of freezing of gait subtypes and the response of each to levodopa in Parkinson's disease. Eur J Neurol 2003;10:391-398.

8. Giladi N, McMahon D, Przedborski S, Flaster E, Guillory S, Kostic V, Fahn S. Motor blocks in Parkinson's disease. Neurology 1992;42:333-339.

9. Giladi N, Kao R, Fahn S. Freezing phenomenon in patients with parkinsonian syndromes. Mov Disord 1997;12:302-305.

10. Stack E, Ashburn A. Fall events described by people with Parkinson's disease: implications for clinical interviewing and the research agenda. Physiother Res Int 1999;4:190-200.

11. Stack EL, Ashburn AM, Jupp KE. Strategies used by people with Parkinson's disease who report difficulty turning. Parkinsonism Relat Disord 2006;12:87-92.

12. Patla AE, Adkin A, Ballard T. Online steering: coordination and control of body center of mass, head and body reorientation. Exp Brain Res 1999;129:629-634.

13. Hausdorff JM, Schaafsma JD, Balash Y, Bartels AL, Gurevich T, Giladi N. Impaired regulation of stride variability in Parkinson's disease subjects with freezing of gait. Exp Brain Res 2003;149: 187-194.

14. Nieuwboer A, Dom R, De Weerdt W, Desloovere K, Fieuws S, Broens-Kaucsik E. Abnormalities of the spatiotemporal character- istics of gait at the onset of freezing in Parkinson's disease. Mov Disord 2001;16:1066-1075.

15. Schaafsma JD, Giladi N, Balash Y, Bartels AL, Gurevich T, Hausdorff JM. Gait dynamics in Parkinson's disease: relationship to Parkinsonian features, falls and response to levodopa. J Neurol Sci 2003;212:47-53

16. Georgiou N, Iansek R, Bradshaw JL, Phillips JG, Mattingley JB, Bradshaw JA. An evaluation of the role of internal cues in the pathogenesis of parkinsonian hypokinesia. Brain 1993;116:11751187.

17. del Olmo MF, Cudeiro J. Temporal variability of gait in Parkinson disease : effects of a rehabilitation programme based on rhythmic sound cues. Parkinsonism Relat Disord 2005;11:25-33.

18. Willems AM, Nieuwboer A, Chavret F, Desloovere K, Dom R, Rochester L, Jones D, et al. The use of rhythmic auditory cues to influence gait in patients with Parkinson's disease, the differential effect for freezers and non-freezers, an explorative study. Disabil Rehabil 2006;28:721-728.

19. Hoehn M, Yahr M. Parkinsonism: onset, progression and mortality. Neurology (Cleveland) 1967;5:427-442.

20. Goetz C, Stebbins GT, Shale HM, Lang AE, Chernik DA, Chmura TA, Ahlskog JE, et al. Utility of an objective dyskinesia rating scale for Parkinson's disease: inter- and intrarater reliability assessment. Mov Disord 1994;9:390-394.

21. Molloy DW, Standish TIM. Mental status and neuropsychological assessment a guide to the standardized mini-mental state examination. Int Psychogeriatr 1997;9:87-94.

22. Giladi N, Shabtai H, Simon ES, Brian S, Tal J, Korczyn AD Construction of freezing of gait questionnaire for patients with Parkinsonism. Parkinsonism Relat Disord 2000;6:165-170.

23. Huxham F, Gong J, Baker R, Morris M, Ianesk R. Defining spatial parameters for non-linear walking. Gait Posture 2006;23:159-163.

24. Taylor MJD, Dabnichki P, Strike SC. A three-dimensional biomechanical comparison between turning strategies during the stance phase of walking. Hum Mov Sci 2005;24:558-573.

25. Thigpen MT, Light KE, Creel GL, Flynn SM. Turning difficulty characteristics of adults aged 65 years or older. Phys Ther 2000; 80:1174-1187.

26. Stack E, Jupp E, Ashburn A. Developing methods to evaluate how people with Parkinson's disease turn $180^{\circ}$ : an activity frequently associated with falls. Disabil Rehabil 2004;26:478-484.

27. Frenkel-Toledo S, Giladi N, Peretz C, Herman T, Gruendlinger L, Hausdorff JM. Effect of gait speed on gait rhythmicity of stride time and swing time respond differently. $J$ neuroeng Rehabil 2005;2:23.

28. Yogev G, Giladi N, Peretz C, Springer S, Simon ES, Hausdorff JM. Dual tasking, gait rhytmicity, and Parkinson's disease: which aspects of gait are attention demanding? Eur J Neurosci 2005;22: $1248-1256$. 
29. Hausdorff JM. Gait variability: methods, modelling and meaning. J Neuroeng Rehabil 2005;2:19.

30. Hausdorff JM, Yogev G, Springer S, Simon ES, Giladi N. Walking is more like catching than tapping: gait in the elderly as a complex cognitive task. Exp Brain Res 164;4:541-548.

31. Zgaljardic DJ, Borod JC, Foldi NS, Mattis P. A review of the cognitive and behavioral sequelae of Parkinson's Disease: relationship to frontostriatal circuitry. Cogn Behav Neurol 2003;16:193210.

32. Morris ME. Movement disorders in people with Parkinson disease: a model for physical therapy. Phys Ther 2000;80:578-597.

33. Debaere F, Wenderoth N, Sunaert S, Van Hecke P, Swinnen SP. Internal vs external generation of movements: differential neural pathways involved in bimanual coordination performed in the presence or absence of augmented visual feedback. Neuroimage 2003; 19:764-776
34. Giladi N, McDermott MP, Fahn S, Przedborski S, Janckovic J, Stern M, Tanner C, and The Parkinson Study Group. Freezing of gait in PD. Prospective assessment in the DATATOP cohort. Neurology 2001;56:1712-1721.

35. Plotnik M, Giladi N, Balash Y, Peretz C, Hausdorff JM. Is freezing of gait in Parkinson's disease related to asymmetric motor function? Ann Neurol 2005;57:656-663.

36. Cubo E, Leurgans SM, Goetz CG. Short-term and practice effects of metronome pacing in Parkinson's disease patients with gait freezing while in the 'on' state: randomized single blind evaluation. Parkinsonism Relat Disord 2004;10:507-510.

37. Orendurff MS, Segal AD, berge JS, Flick KC, Spanier D, Klute GK. The kinematics and kinetics of turning: limb asymmetries associated with walking a circular path. Gait Posture 2006;23:112117. 